\title{
Liquid-crystal point-diffraction interferometer for wave-front measurements
}

\author{
Carolyn R. Mercer and Katherine Creath
}

\begin{abstract}
A new instrument, the liquid-crystal point-diffraction interferometer $\mid$ LCPDI , is developed for the measurement of phase objects. This instrument maintains the compact, robust design of Linnik's point-diffraction interferometer and adds to it a phase-stepping capability for quantitative interferogram analysis. The result is a compact, simple to align, environmentally insensitive interferometer capable of accurately measuring optical wave fronts with very high data density and with automated data reduction. We describe the theory and design of the LCPDI. A focus shift was measured with the LCPDI, and the results are compared with theoretical results.

Key words: Phase-stepping interferometry, wave-front measurement, common-path interferometer, error reduction, phase-measurement algorithm. 1996 Optical Society of America
\end{abstract}

\section{Introduction}

The point-diffraction interferometer (PDI) has long been used for the measurement of optical wave fronts for lens testing and fluid flow diagnostics. It was invented by Linnik ${ }^{1}$ and reinvented by Smartt and co-workers. ${ }^{2,3}$ Linnik's original paper is in Russian; an English translation is included in a paper by Speer et al. ${ }^{4}$ The PDI's primary advantage is its common-path design. An interferogram can be formed with only a single laser path rather than with the two paths required for Mach-Zehnder or Michelson interferometers. This is especially important in the measurement of large objects such as wind tunnel flows, in which the optical paths are very long and air turbulence must be minimized along the paths. A single path is also advantageous when the size of the instrument must be kept small. The common-path design requires relatively few optical elements, thereby reducing the cost, size, and weight of the instrument and simplifying alignment. Although the PDI is a common-path interferometer, air turbulence in the object beam will affect the results, as will relative motion of the filter relative to the optical train. Therefore care must still be taken to provide a stable environment for operating the PDI.

C. Mercer is with NASA Lewis Research Center, Cleveland, Ohio 44135. K. Creath is with the Optical Sciences Center, University of Arizona, Tueson, Arizona 85716

Received 7 June 1995 ; revised manuscript received 10 November 1995 .

0003-6935 96 101633-10\$06.00 0

c) 1996 Optical Society of America
The PDI has been used to test a variety of optical elements, ${ }^{4,5}$ and its simple alignment makes it useful for optical testing in the IR, ${ }^{6} \mathrm{UV}$, and $\mathrm{x}$-ray ${ }^{7}$ spectral regions. Although Linnik ${ }^{1}$ implied in the 1930's that the PDI would be useful for fluid studies, it was not until relatively recently that this application was demonstrated. ${ }^{8}$ The PDI is currently being evaluated for use in microgravity studies aboard the Space Shuttle. , $^{9} 10$

Like any interferometer, the interferograms produced by the PDI must be interpreted to extract information about the object wave front. The most accurate and effective way to measure both the magnitude and the sign of wave-front aberrations is to use phase-shifting interferometry. ${ }^{11}$ The PDI has traditionally been unable to use this advanced technique, however, because its common-path design makes it difficult to shift the phase of one beam relative to the other.

Several modifications have been proposed to add phase shifting to the PDI. Underwood et al. ${ }^{12}$ added phase stepping to a refiection PDI. They forced the object and reference beams to have orthogonal polarizations, and they used an electro-optic modulator to phase shift one relative to the other. The system used several extra optical elements and had a very low optical throughput efficiency. A variation of the PDI developed by Kwon ${ }^{13}$ used a specially fabricated diffraction grating to produce three phase-shifted interferograms simultaneously. The wave-front phase can be determined from these interferograms, but three area detectors are required and the relative phase is fixed once the grating has been fabri- 
cated. This device is useful for high-speed applications because time delays are not required between the acquisition of each interferogram. Kadono et $a l{ }^{14}$ added phase stepping to the PDI by using polarizing optics. The technique was demonstrated to have an accuracy of $\lambda / 40$, but it is limited to the measurement of small objects or very slow lenses because the incident light must be nearly normal to the polarizer.

A new version of the Zernike interferometer, ${ }^{15}$ similar to the PDI, was recently developed by Kadono et al. ${ }^{16}$ Their device is very similar to the liquid-crystal PDI described in this paper. Both instruments use a thin liquid-crystal layer to phase shift one beam relative to another, but they differ in the generation of the reference beam. Kadano et al. use a circle etched in the electrodes to prevent molecular rotation in a cylindrical region nominally $100 \mu \mathrm{m}$ in diameter. The relatively large size of the hole causes their device to act as a Zernike phasecontrast interferometer rather than a PDI. The lower limit on their hole diameter is limited by electric-field ringing across the edges; they indicate that the ringing extends in $\sim 10 \mu \mathrm{m}$, permitting a minimum hole diameter of $\sim 20 \mu \mathrm{m}$.

Common-path phase-shifting interferometers have also been developed by Iwata and Nishikawa ${ }^{17}$ and by Churin and Sedukhin. ${ }^{18}$ Iwata's device is for the measurement of opaque surfaces, and Churin's is limited to the measurement of planar phase objects. We fully describe a new interferometer, the liquidcrystal point-diffraction interferometer ${ }^{19}$ (LCPDI), based on the classic PDI. The LCPDI employs a liquid-crystal layer to introduce arbitrary phase shifts between the object and reference beams. A microsphere embedded within the liquid-crystal layer provides a locally generated reference beam. The object beam is phase shifted by modulation of the voltage across the liquid crystals, which alters the refractive index of the birefringent nematic liquid crystals. This permits completely flexible phasestepping interferometry capability while retaining the fully common-path optical design.

\section{Physical Description}

The principle of the LCPDI is the same as that of the classic PDI; the differences lie in the construction of the filters. The PDI uses a neutral-density filter with a pinhole, whereas the LCPDI uses a liquidcrystal layer with an embedded microsphere. In both cases, light is first reflected off or transmitted through an object of interest, and then brought to a focus on the diffracting element. The diameter of this element is smaller than the focused spot, and so a spherical wave is generated by diffraction. The portion of the incident light unaffected by the diffracting element is transmitted through and attenuated by the filter. Information contained in the incident wave is retained in the attenuated beam but filtered out of the diffracted wave. The two components of the transmitted wave are therefore referred to as the object and reference waves, respectively. They both travel coincidentally behind the filter, and when combined coherently produce an interferogram whose appearance depends on the information in the incident wave of light. Good contrast is achieved by attenuation of the object beam such that the relative intensities of the object and reference beams are similar.

The LCPDI is shown schematically in Fig. 1. Merck E7 nematic liquid crystals (LC's) are sandwiched between two glass plates (G's), each nominally $0.5 \mu \mathrm{m}$ thick and $3.0 \mathrm{~cm} \times 3.5 \mathrm{~cm}$ across. Cylindrical rods (R's) with a diameter of $9 \mu \mathrm{m}$ are placed at the edges of the plates to serve as spacers. Transparent plastic microspheres (M's), nominally 9 $\mu \mathrm{m}$ in diameter, are scattered throughout the liquidcrystal layer. Each microsphere replaces a small volume of liquid crystals as the filler between the glass plates. Transparent electrodes (E's) are deposited on plates' inner surfaces, and leads (L's) are soldered onto the electrodes so that an alternating current can be applied across the liquid-crystal layer.

The glass plates in the LCPDI are prepared so that the birefringent liquid crystals are homogeneously aligned with their directors (long axes) oriented horizontally, parallel to the plates. This configuration allows phase modulation of horizontally polarized light traveling through the layer. ${ }^{20}$ Vertically polarized light will not be phase shifted. The uniaxial liquid-crystal layer has a refractive index equal to the extraordinary refractive index, $n_{e}$, for light polarized parallel to the aligned directors. As the amplitude of the applied electric field increases, the molecules rotate, and the refractive index of the layer shifts toward the ordinary index of refraction. The shift occurs until the directors are perpendicular to the glass plates and the refractive index equals the ordinary index (Fig. 2).

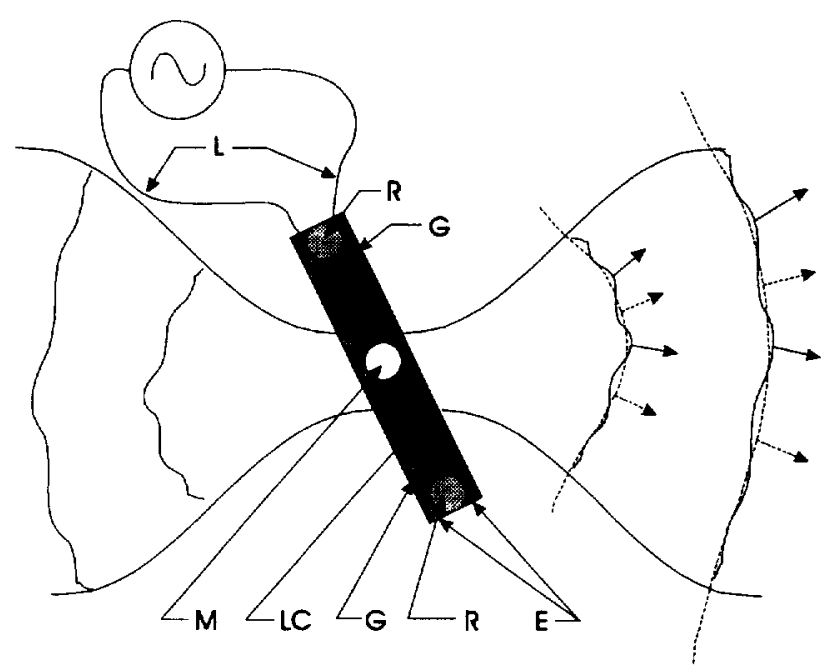

Fig. 1. Schematic of the LCPDI showing the liquid-crystal layer, glass plates, microsphere, spacing rods, electrodes, and leads. The object wave is shown as a solid curve and the reference wave is shown as a dashed curve. 

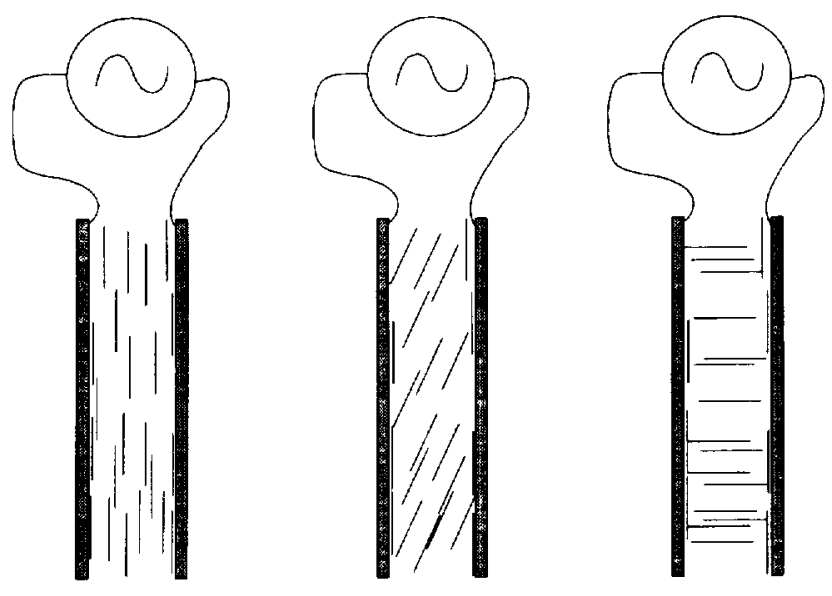

$$
V<V_{m}
$$

$V_{m}<V<V_{\text {sot }}$

$V_{\text {or }}<V$

Fig. 2. Effect of applied electric field on liquid-crystal molecules.

The difference between the ordinary and extraordinary refractive indices of Merck E7 is $\Delta n=0.2414$ at $514.5 \mathrm{~nm}$ and $20^{\circ} \mathrm{C}$. This large birefringence permits a $2 \pi$ phase change, using a very thin liquidcrystal layer. If edge effects ${ }^{21}$ were not important, a 4.3- $\mu \mathrm{m}$ layer would permit a full $4 \pi$ phase change, allowing the use of six-step phase-extraction algorithms. We used spacing rods and microspheres nominally $9 \mu \mathrm{m}$ in diameter to provide a $9-\mu \mathrm{m}$ liquid-crystal layer, leaving a comfortable safety margin. The refractive index of the liquid crystals depends roughly linearly on temperature, with a slope of approximately $-0.0014{ }^{\circ} \mathrm{C}^{-1}$ over the range $0-38{ }^{\circ} \mathrm{C}$. For $\Delta n$ to be kept within $1 \%$ of its value, the temperature of the LCPDI should be maintained within $2{ }^{\circ} \mathrm{C}$.

Dye added to the liquid crystals attenuates the object beam to roughly the same intensity as the reference beam. The dye's optical density is 1.4 when the dye molecules are oriented parallel to the incident light polarization. The dye improves the fringe contrast, but the dye molecules rotate with the liquid-crystal molecules, causing an unwanted intensity modulation when the phase is shifted. ${ }^{22}$ The consequences of this are discussed in Subsection 3.B.

The LCPDI is tilted about the horizontal axis to minimize secondary fringes caused by multiple reflections from the glass plates. Antireflection coatings on each glass surface can be used in place of this tilt. The LCPDI itself introduces aberrations into the interferograms because the filter is a plane-parallel plate placed in a converging beam. These aberrations must be subtracted from the measured wave front. This correction will occur automatically if the device is used to measure an initial wave front subtracted from an altered wave front. This is usually the case in fluid-studies measurements.

Care must be taken to ensure that only a single microsphere is illuminated by the incident light because all microspheres will generate an interference pattern. These multiple patterns will also phase shift, producing measurement errors.
Although nematic liquid crystals have published response times of $10-100 \mathrm{~ms}$, these values are limited by the time required to restore the liquidcrystal molecules to their relaxed state. This consideration is unimportant for the LCPDI because the required switching is from one intermediate state to another. Electrically driving the molecular orientation is much faster than waiting for full relaxation; typical response times for this process are only $0.5-2$ $\mathrm{ms}$ and perhaps as fast as $10-50 \mu \mathrm{s}^{23}$

The electric-field amplitudes required for $90^{\circ}$ phase shifts were determined by placement of a pointer on a fringe boundary on the ground-glass screen. The intensity at the pointer was observed as the amplitude was varied across the liquid-crystal layer. A sequence of $1.04,1.25,1.35,1.45$, and $1.55 \mathrm{~V} \mathrm{rms}$ produced five $90^{\circ}$ phase shifts. The first voltage setting is not critical; it merely has to be at or below the threshold voltage that causes the Freedericksz transition of the liquid crystals.

The absorptance of the liquid-crystal layer was calibrated when a horizontally polarized collimated beam was passed through the LCPDI and the transmitted intensity was recorded as the voltage was varied across the electrodes. The LCPDI was tilted by $27^{\circ}$ relative to the optic axis, and the relaxed liquid-crystal molecules were parallel to the incident polarization. The intensity as a function of applied voltage is plotted in Fig. 3. The plot shows that the absorption of the dye varies strongly with the orientation of the liquid crystals, with the attenuation decreasing as the molecules are rotated farther from their initial state. This behavior is the same as that observed by Heilmeier et $a{ }^{24}$; they demonstrated that the attenuation variation is caused by asymmetric dye molecules rotating with the liquid crystals.

The variation shown in the plot is nonlinear but is repeatable; two sequences of 100 measurements are shown superimposed on each other. From this plot, the relative intensity of the object beam at each applied voltage can be determined. For the five voltages listed above, the relative intensities are as

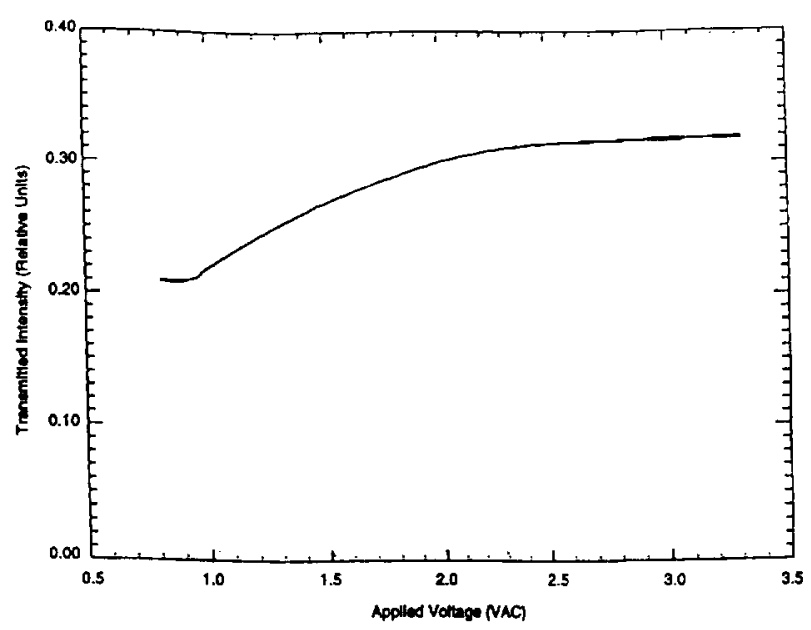

Fig. 3. Liquid-crystal layer intensity calibration curve. 
follows: $0.21,0.59,0.74,0.88$, and 1.00 , where the $\mathrm{dc}$ level is considered to be zero.

\section{Theory}

A. Principle of the LCPDI

\section{Ray Model}

A ray model of the LCPDI is shown in Fig. 4. The LCPDI filter is represented by a liquid-crystal layer of thickness $t$ sandwiched between two glass plates of thickness $t_{g}$ and refractive index $n_{g}$. The object beam is modeled as rays converging at a single point within the filter; the reference beam is modeled as a ray fan originating from this point. A point on the surface of the liquid-crystal layer was chosen instead of the midpoint to simplify the equations; this choice does not fundamentally affect the results of the model. The object beam travels through the liquidcrystal layer with refractive index $n_{\mathrm{LC}}$, and the reference beam travels through the microsphere (not shown) with index $n_{\text {sphere. }}$. The phase difference, $\Delta \phi$, between the waves represented by these rays in a plane $r_{2}$ behind the LCPDI is given by

$$
\begin{aligned}
\Delta \phi(\alpha)= & \left.\phi_{\mathrm{obj}}(\alpha)+(2 \pi / \lambda) \mid t_{g} n_{g} / \cos \left(\sin ^{-1}\left(\sin \alpha / n_{g}\right)\right]\right\} \\
& \left.+(2 \pi / \lambda) \mid t\left(n_{\mathrm{LC}}-n_{\text {sphere }}\right) / \cos \left[\sin ^{-1}\left(\sin \alpha / n_{\mathrm{LC}}\right)\right]\right\}
\end{aligned}
$$

where $\phi_{o b j}$ represents the phase distribution of the object rays at plane $r_{1}, \alpha$ is the angle the particular ray makes with the optic axis, and $\lambda$ is the wavelength of the incident light. In general, this phase difference is not the constant across the wave front because the beam is either converging or diverging through the layer, but in practice this effect is small and the small-angle approximation can be made. ${ }^{25}$ Therefore Eq. (1) can be approximated by

$$
\Delta \phi(\alpha) \cong \phi_{\mathrm{obj}}(\alpha)+\phi_{0}+(2 \pi / \lambda) t\left(n_{\mathrm{LC}}-n_{\text {sphere }}\right),
$$

where $\phi_{0}$ is a fixed phase delay between the two waves.

This expression relates the phase distribution in a plane behind the LCPDI to the phase distribution of

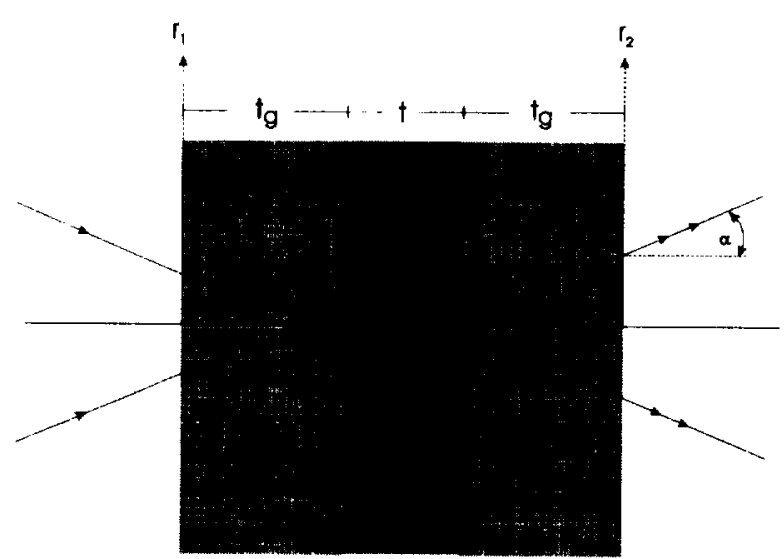

Fig. 4. Ray model of the LCPDI. the original object wave. It can be seen that the original information is retained with the addition of only a simple piston shift. This shift can be stepped or ramped by variation of the refractive index of the liquid-crystal layer.

\section{Wave Model}

Fourier theory can be used to analyze the LCPDI. Figure 5 shows a system schematic that indicates the planes used in the following analysis. The interferogram is imaged onto a camera, and the object and reference waves in the image plane are determined by the use of Fourier analysis. A unitamplitude plane wave, with wavelength $\lambda$ and aberration $W\left(r_{1}\right)$, is incident upon an aberration-free thin lens $L_{1}$ having diameter $D$ and focal length $f$. The optical field just behind $L_{1}$ is described by the product of the initial wave and the lens pupil function:

$$
u_{1}\left(r_{1}\right)=\operatorname{cyl}\left(r_{1} / D\right) \exp \left[(2 \pi i / \lambda) W\left(r_{1}\right)\right] .
$$

The cylinder function, cyl $\left(r_{1} / D\right)$, represents a function equal to zero everywhere except for $r_{1}<D / 2$, where the function equals one. ${ }^{26}$ This field propagates to the focal plane, where it is described by its Fourier transform, $U_{1}\left(r_{2}\right)$, where the transform is evaluated at the spatial frequency $\rho=r_{2} / \lambda f$. At the focal plane the field is multiplied by the transmittance of the PDI plate $\tau\left(r_{2}\right)$, given by

$$
\begin{aligned}
\tau\left(r_{2}\right)= & {\left[\tau_{b}+\left(1-\tau_{b}\right) \operatorname{cyl}\left(r_{2} / d\right)\right] } \\
& \times \exp \left(i\left[\phi_{b}+\left(\phi_{0}-\phi_{b}\right) \operatorname{cyl}\left(r_{2} / d\right)\right],\right.
\end{aligned}
$$

where $\tau_{b}$ and $\phi_{b}$ are the transmittance of and phase delay caused by the background plate, $d$ is the microsphere diameter, and $\phi_{0}$ is the phase delay of the microsphere. The microsphere transmittance is unity. This plate is shown graphically in Fig. 6. Although a microsphere is used in this filter, it is equivalent to use of a hole or a spot to form the diffracted reference beam because of Babinet's principle. A microsphere was used because it is easier to embed a very small sphere in a liquid-crystal layer than to produce a very small hole in it.

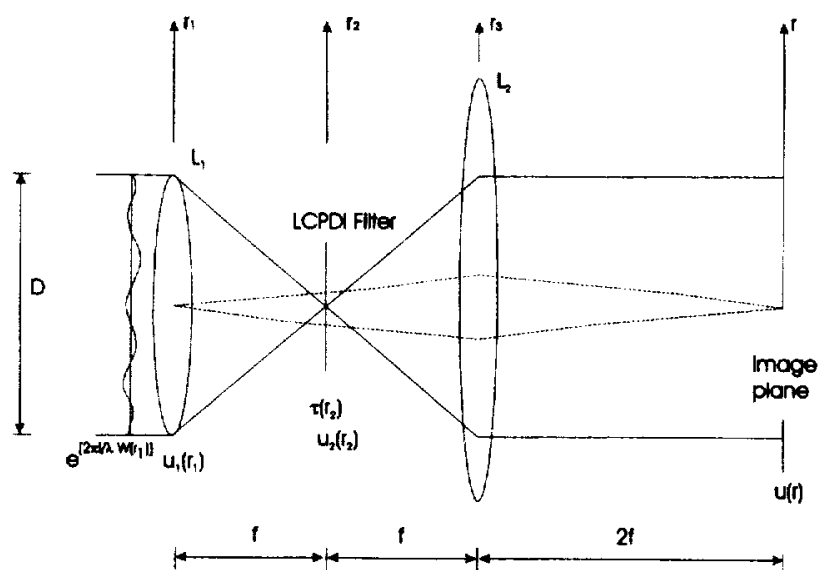

Fig. 5. Planes used in Fourier analysis. 

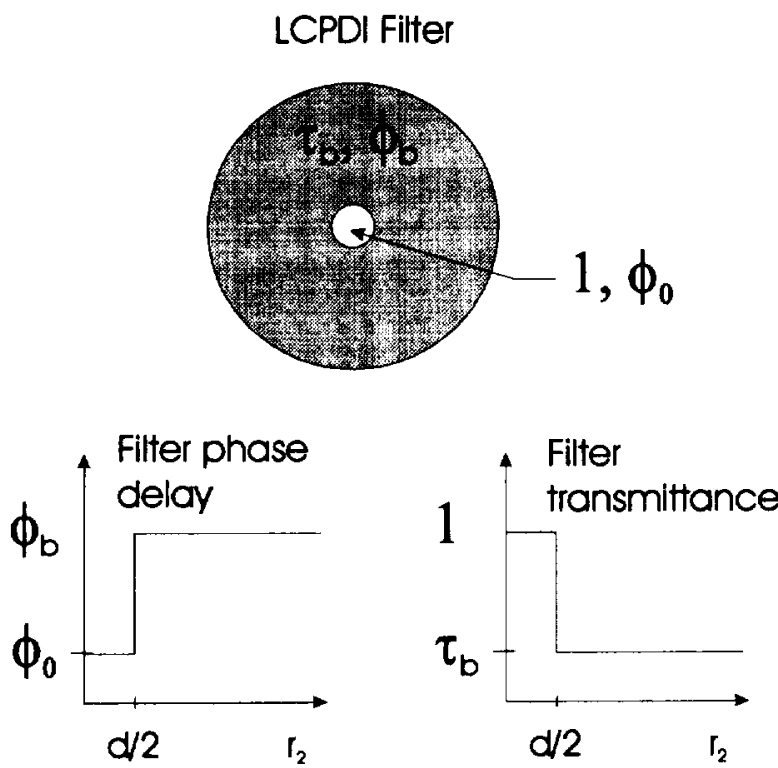

Fig. 6. Transmittance and phase delay of the LCPDI filter.

The optical field just behind the pinhole is described by

$$
u_{2}\left(r_{2}\right)=\tau\left(r_{2}\right) U_{1}\left(r_{2}\right)
$$

Lens $L_{2}$ images the field at plane 1 through the filter and onto the image plane. The effect of the filter can be understood by consideration of an effective wave front emerging from lens $L_{1}$ and passing through no filter. For the known wave front just behind the focal plane given by Eq. (5) to be created, the effective wave front behind $L_{1}$ must equal the inverse Fourier transform of $u_{2}$. The image then becomes a scaled copy of this effective wave convolved with the imaging lens impulse response function. For a high quality, thin spherical lens used paraxially, this function is a sombrero function ${ }^{26}$ that smooths the image; for the purpose of this discussion it is assumed to be a delta function. This is the limiting form of the sombrero function as the lens diameter becomes very large and the lens becomes very thin. Assuming unit magnification, a focused beam centered on the pinhole, and neglecting the Petzval curvature across the image and a complex proportionality constant, one can describe the field in the image plane by

$$
u(r)=T(r) * * u_{1}(r)
$$

where the double asterisk represents a two-dimensional convolution in circular coordinates, and $T(r)$ is the Fourier transform of filter function $\tau\left(r_{2}\right)$, given by

$$
\begin{aligned}
T(r)= & \left.\mid \exp \left(i \phi_{0}\right)-\tau_{b} \exp \left(i \phi_{b}\right)\right]\left|\left(\pi d^{2}\right) / 4 \lambda f\right| \operatorname{somb}(d r / \lambda f) \\
& +\tau_{b} \exp \left(i \phi_{b} \mid \delta(r / \lambda f) / \pi r,\right.
\end{aligned}
$$

where $\delta($ ) is the one-dimensional Dirac delta function; the sombrero function sombicr is given by $2 J_{1}(\pi c r) /(\pi c r)$, where $c$ is a constant and $J_{1}(x)$ is a first-order Bessel function of the first kind.
The optical field at the image plane is then ${ }^{25}$

$$
\begin{aligned}
u(r)= & \left.\tau_{b} \exp \left(i \phi_{b}\right) \operatorname{cyl}(r / D) \exp \mid(2 \pi i / \lambda) W(r)\right] \\
& +(1 / \lambda f)\left[\exp \left(i \phi_{0}\right)-\tau_{b} \exp \left(i \phi_{b}\right) \mid \pi d^{2} 4 \lambda f\right) \\
& \times \operatorname{somb}(d r / \lambda f) * * \operatorname{cyl}(r / D) \exp [(2 \pi i \lambda) W(r)
\end{aligned}
$$

where the complex constant of proportionality has been dropped. Equation (8) shows that the interferogram is formed by the coherent addition of the attenuated object wave front and a reference wave front formed by the convolution of the object wave front with the Fourier transform of the LCPDI plate, corresponding to the first and second terms in this equation, respectively. The reference wave front is a smoothed version of the object wave, and the phase difference between the object and reference waves can be varied by the control of either $\phi_{0}$ or $\phi_{b}$.

In the limit as the pinhole diameter approaches zero, the sombrero function approaches unity, and the reference wave becomes an integrated version of the object wave, i.e., a smooth continuous function. Note that this is not independent of the object wave, but for relatively slowly varying functions $W(r)$ the reference wave becomes spherical. Figure 7 shows the effect of the pinhole size on the generated reference wave. A cross section of the original, computer-simulated wave front is shown in plot (a), followed by plots (b) through $(\mathrm{h})$, which show corresponding cross sections in the image plane of reference beams calculated with pinholes of increasing size. The pinhole diameters in these plots are equal to $0.1,1,3,10,33,100$, and 1000 times the Airy disk radius, respectively. In each of the latter plots, the portion of the focused beam intersected by the pinhole is shown on the left, and the reference beam generated by that pinhole is shown on the right. The abscissa is scaled by lens diameter $D$, but the focused beam plot is magnified in the $x$ axis by $D d d$ for clarity.

Figure 7 shows that even a pinhole one tenth the size of the Airy disk radius [Fig. 7(b) does not generate a spherical wave when the object wave is highly aspheric. A pinhole three times larger than the Airy disk radius [Fig. 7(d)] still filters out most of the object wave, but the object wave structure is noticeable at ten times $[\mathrm{Fig}$. 7(e)], and pronounced at 33 times [Fig. 7(f)] the recommended size. By the time the pinhole is 1000 times the Airy disk radius [Fig. $7(\mathrm{~h})$ ), the entire object wave is transmitted. The correct size for the diffracting element depends on the spatial frequency content in the object wave. For highly irregular object waves such as those created by some fluids experiments, a diffracting element with a diameter of approximately three times larger than the Airy disk radius is acceptable. For nearly spherical waves such as those created by high-quality optical elements, the diffracting element should be kept smaller than one Airy disk 
(a) Original Wave front

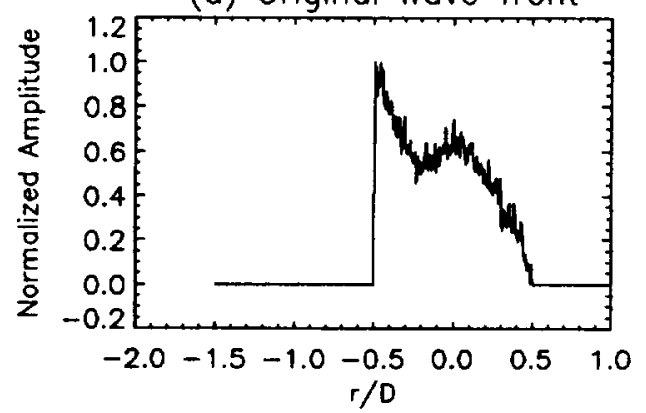

(c) $d=$ (1.22 lambdo fNo)

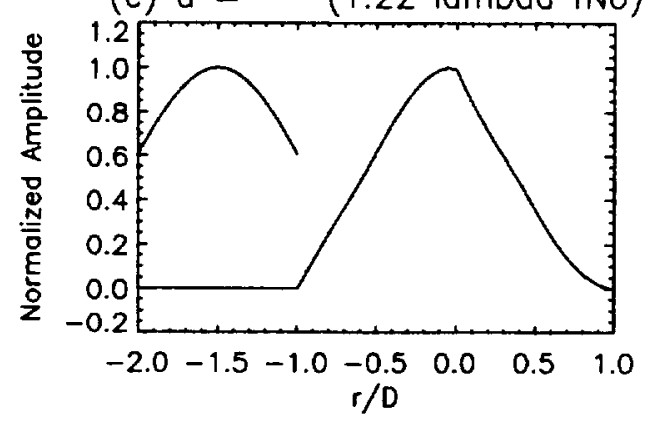

(e) $d=10(1.22$ lambdo $\mathrm{fNo})$
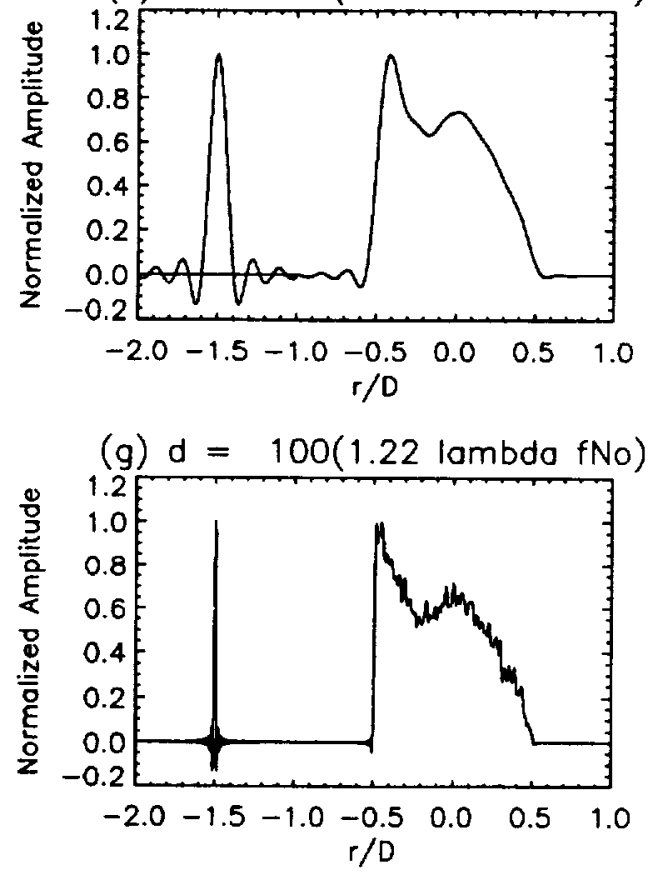

(b) $d=0.1$ (1.22 lambda fNo)
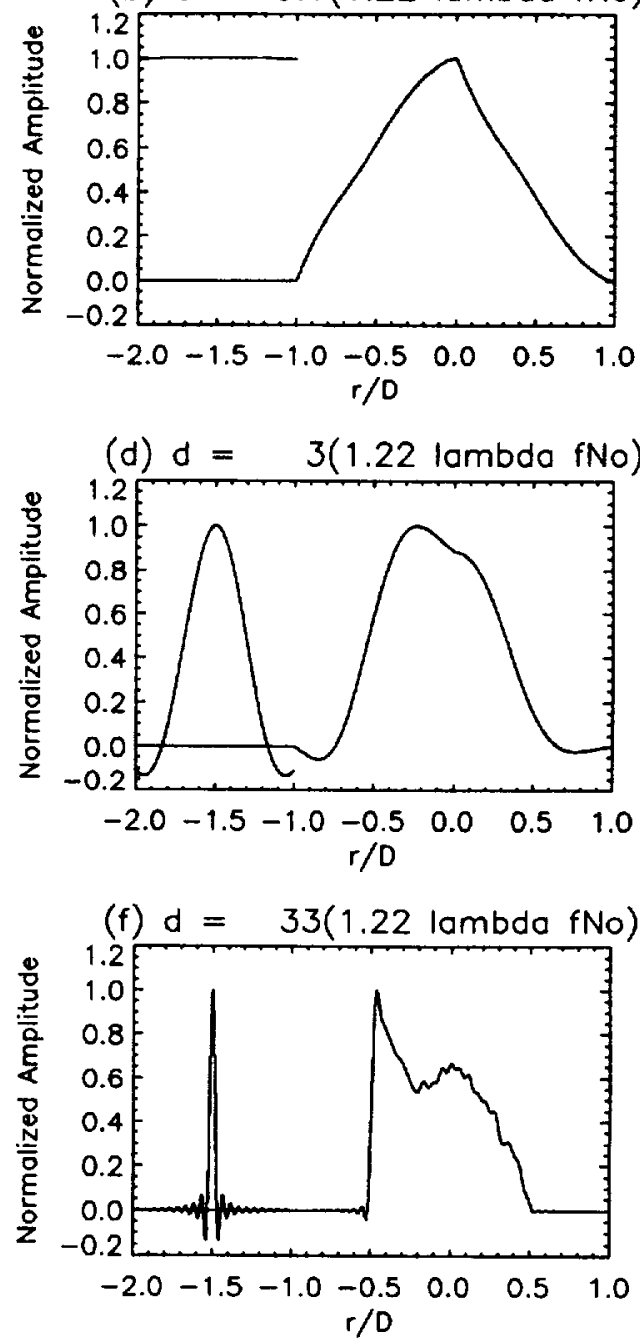

(h) $d=1000(1.22$ lambda fNo)

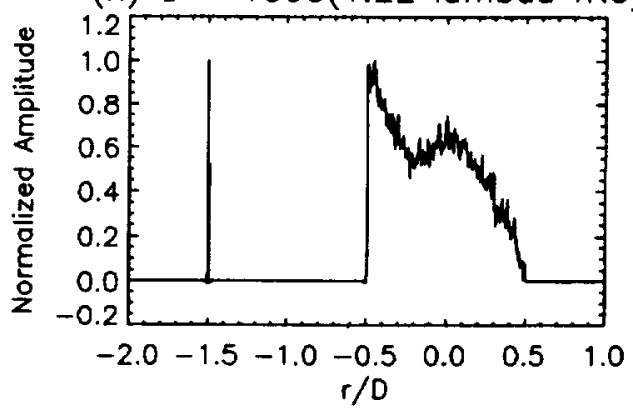

Fig. 7. Effect of pinhole diameter on reference wave.

radius. ${ }^{25}$ Koliopoulos et al. . recommend a pinhole diameter less than or equal to one half the Airy disk diameter. ${ }^{6}$ Note also that the aperture used for the Zernike interferometer described by Kadono et al. ${ }^{16}$ is approximately 33 times the Airy disk radius. Clearly only the high-frequency content of the object wave can be discriminated by this method. The LCPDI uses a 9- $\mu \mathrm{m}$ microsphere, and the data presented in this paper were obtained with an $f / 6$ beam. This results in a diffracting element three times the Airy disk radius, corresponding to Fig. 7(d).

\section{B. Phase Calculation}

There are two assumptions that must be satisfied to use standard phase-extraction algorithms for phaseshifting interferometry. One assumption is that the phase steps must be uniform across the interfero- 
gram; the other is that neither the object nor the reference beam intensities vary from frame to frame.

Errors caused by the nonuniform phase shifts manifest themselves as a phase-measurement error that occurs at twice the spatial frequency of the interference fringes. Although the LCPDI shifts the phase of a convergent beam, this error source is negligible and can be ignored. ${ }^{25}$ Errors caused by the intensity variations appear as phase errors occurring at the same frequency as the fringes. This error is the dominant source of error in the LCPDI. This effect is caused by rotation of the dye molecules, and the variation is substantial. Therefore, the standard algorithms must be modified to reduce these errors.

If one can determine the object beam intensity independently, then the algorithms can be modified in two ways. The first is for one to solve for the phase by using the object beam intensity distributions explicitly; the second is for one simply to normalize each interferogram by its corresponding object beam intensity distribution. The latter method works best if the object beam intensity is much stronger than the reference beam.

The first way to calculate wave-front phase $\phi$ is to use the following equation:

$$
\begin{aligned}
\tan (\phi)= & \left(\frac{\Delta I_{3}-\Delta I_{1}}{\Delta I_{0}+\Delta I_{4}-2 \Delta I_{2}}\right) \\
& \times\left(\frac{\sqrt{I_{0}{ }^{\mathrm{obj}}}+\sqrt{I_{4}{ }^{\mathrm{obj}}}+2 \sqrt{I_{2}{ }^{\mathrm{obj}}}}{\sqrt{I_{3}{ }^{\mathrm{obj}}}+\sqrt{I_{1}{ }^{\mathrm{obj}}}}\right),
\end{aligned}
$$

where $I_{j}{ }^{\text {obj }}$ is the $j$ th object beam intensity distribution, $\Delta I_{j}=I_{j}-I_{j}{ }^{\mathrm{bbj}}, I_{j}$ is the $j$ th interferogram intensity distribution, and the explicit pixel dependency $(x, y)$ has been dropped for brevity. This equation is exact, provided that the reference beam intensity remains constant from frame to frame.

For the second compensation method, each interferogram $I_{j}$ is divided by the appropriate normalization frame and then used in the five-frame algorithm as follows:

$$
\tan (\phi)=\frac{2\left(I_{3} / I_{3}{ }^{\mathrm{obj}}-I_{1} / I^{\mathrm{obj}}\right)}{\left(I_{0} / I_{0}{ }^{\mathrm{obj}}+I_{4} / I_{4}{ }^{\mathrm{obj}}-2 I_{2} / I_{2}{ }^{\mathrm{obj}}\right)} .
$$

This equation is not exact and works best if $I_{j}^{\text {obj }} \gg$ $I_{j}^{\text {ref }}$, so that $I_{j}^{\mathrm{obj}}+I_{j}^{\text {ref }}$ is approximately equal to $I_{j}^{\mathrm{obj}}$.

In either case the intensity distribution of the object beam alone must be determined. This is not trivial because the object and reference beams are difficult to separate in a common-path interferometer. There are four ways that this information can be obtained. The first is to obtain the peak values of the object beams at each phase step from an intensity calibration curve, such as the one in Fig. 3. The shape of the distribution must be estimated, though; a Gaussian distribution is a reasonable assumption, but there is little information available for choosing the Gaussian width.

The second way to get the object beam intensity is to translate the LCPDI perpendicularly to the optic axis to let the focused beam pass through the instrument without hitting a microsphere. No reference beam will be generated, so the light incident upon the viewing screen is from the object beam alone. This light can be recorded for each of the phase steps.

The third way is to fit two-dimensional polynomials to the object beam distributions obtained by translation of the LCPDI, rather than by the direct use of the recorded intensity distributions. This effectively smooths out speckles and imperfections in the viewing screen.

The final way to get the data is to fit a polynomial to each interferogram, rather than to each object beam distribution. This yields the average intensity across the detector: the incoherent sum between the object and reference beams. This estimate is more appropriate for use in Eq. (10) than in the exact equation, and it is especially appropriate when the object beam is substantially stronger than the reference beam. This technique has the advantage that no calibration is necessary, the LCPDI need not be moved, and fewer frames of data are required.

\section{Performance}

The accuracy of the LCPDI was tested when a known phase object was measured with the LCPDI and the measured data were compared with expected results. The difference between two wave fronts was measured rather than an individual wave front. This wave-front difference was generated when the focus of the optical system was shifted, and the expected results were calculated from theory.

\section{A. Focus Shift Measurement}

Figure 8 shows an expanded view of the optical system near the LCPDI filter. A 2 -mW, collimated, 514.5-nm laser beam was passed through a $100-\mathrm{mm}$ Cooke triplet lens $\left(\mathrm{L}_{1}\right)$ stopped down to $/ 6.2$. The filter was mounted on a three-axis positioner placed near the focused spot and finely positioned to get a good fringe pattern. A horizontally oriented linear polarizer (P2) was placed behind the filter to remove depolarized light scattered from the liquid-crystal molecules. A ground glass-screen (SCR) placed 40 $\mathrm{cm}$ behind the focal spot rendered the interferogram

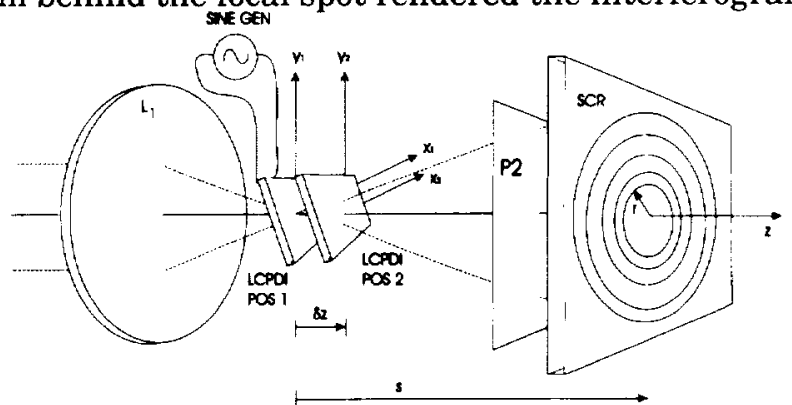

Fig. 8. Optical configuration for defocus measurement. 
visible, and a $50-\mathrm{mm} f / 2.8$ lens imaged the interferogram onto a $640 \times 480$ pixel, 8-bit CCD camera. The detector plane was $66 \mathrm{~cm}$ behind the groundglass screen, and the LCPDI was tilted by $\sim 20^{\circ}$ to decrease multiple interference effects off the uncoated glass surfaces. Phase shifts of $\pi / 2 \mathrm{rad}$ were added to the object beam by application of a voltage sequence of $1.04,1.25,1.35,1.45$, and $1.55 \mathrm{~V}$ rms. The interferogram produced after each phase shift was recorded by the camera and then acquired and stored on a personal computer.

To compensate for intensity variations caused by the rotating dye molecules, the intensity distribution of the object beam alone was measured by translation of the LCPDI plate by $0.75 \mathrm{~mm}$ along the $x_{1}$ axis so that the focused beam did not pass through any microspheres. The light incident upon the screen was recorded for each applied voltage. The amount of defocus was then increased when the LCPDI was moved along the optical axis by $\delta z=0.34 \mathrm{~mm}$. Slight in-plane adjustments were made to center the new interference pattern on the CCD. Again, the interferograms were recorded, and the LCPDI plate was translated to record the object beam alone. This amount of defocus changed the beam diameter on the plate from roughly 18 to $-23 \mu \mathrm{m}$, which is roughly a $30 \%$ change. The fringe contrast was, however, acceptable at both positions.

The wave fronts for both positions of the LCPDI were computed by the use of Hariharan's standard five-frame algorithm on the raw interferograms. The wave-front difference was obtained by subtrac- tion of the two calculated wave fronts. Two-dimensional polynomials were fitted to the two calculated wave fronts, and the difference between these two polynomials was also found. This difference shows the underlying shape of the measured wave-front difference.

Each frame of object beam intensity data was smoothed with a boxcar average over 25 pixels to remove high spatial frequency information from the frames. A two-dimensional sixth-order polynomial was fitted to each smoothed intensity frame to form the normalization frames $I_{j}$ obj. The wave fronts at both LCPDI positions were calculated by the use of both Eqs. (9) and (10), and the differences at each position were calculated.

\section{B. Theoretical Calculation of Focus Shift}

The expected difference in the optical phase between the wave fronts at each LCPDI position was calculated from

$$
\begin{aligned}
\Delta \phi_{\mathrm{th}}(r, \delta z)= & (2 \pi / \lambda)\left(\left(s^{2}+r^{2}\right)^{12}\right. \\
& \left.\left.-\left\{(s-\delta z)^{2}+r^{2}\right]\right\}^{12}-\delta z\right),
\end{aligned}
$$

where $s$ is the distance from the LCPDI to the ground-glass viewing screen, $\delta z$ is the axial distance between the two positions of the LCPDI, and $r$ is the radial distance from the center of the interferogram.

\section{Results}

Figure 9 shows the wave front measured with the LCPDI at position 2, computed with the standard

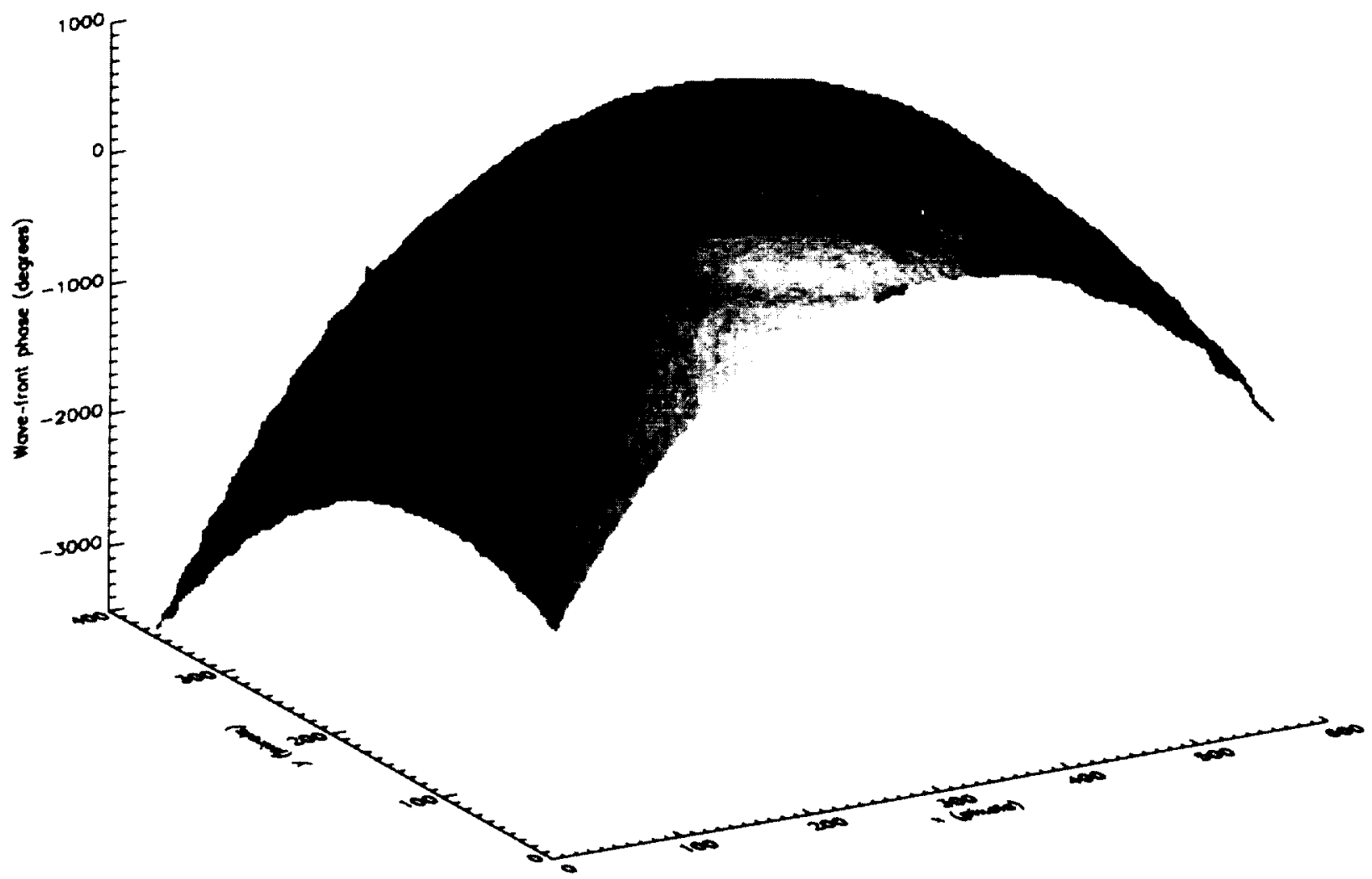

Fig. 9. Wave-front difference calculated with a standard five-frame algorithm. 


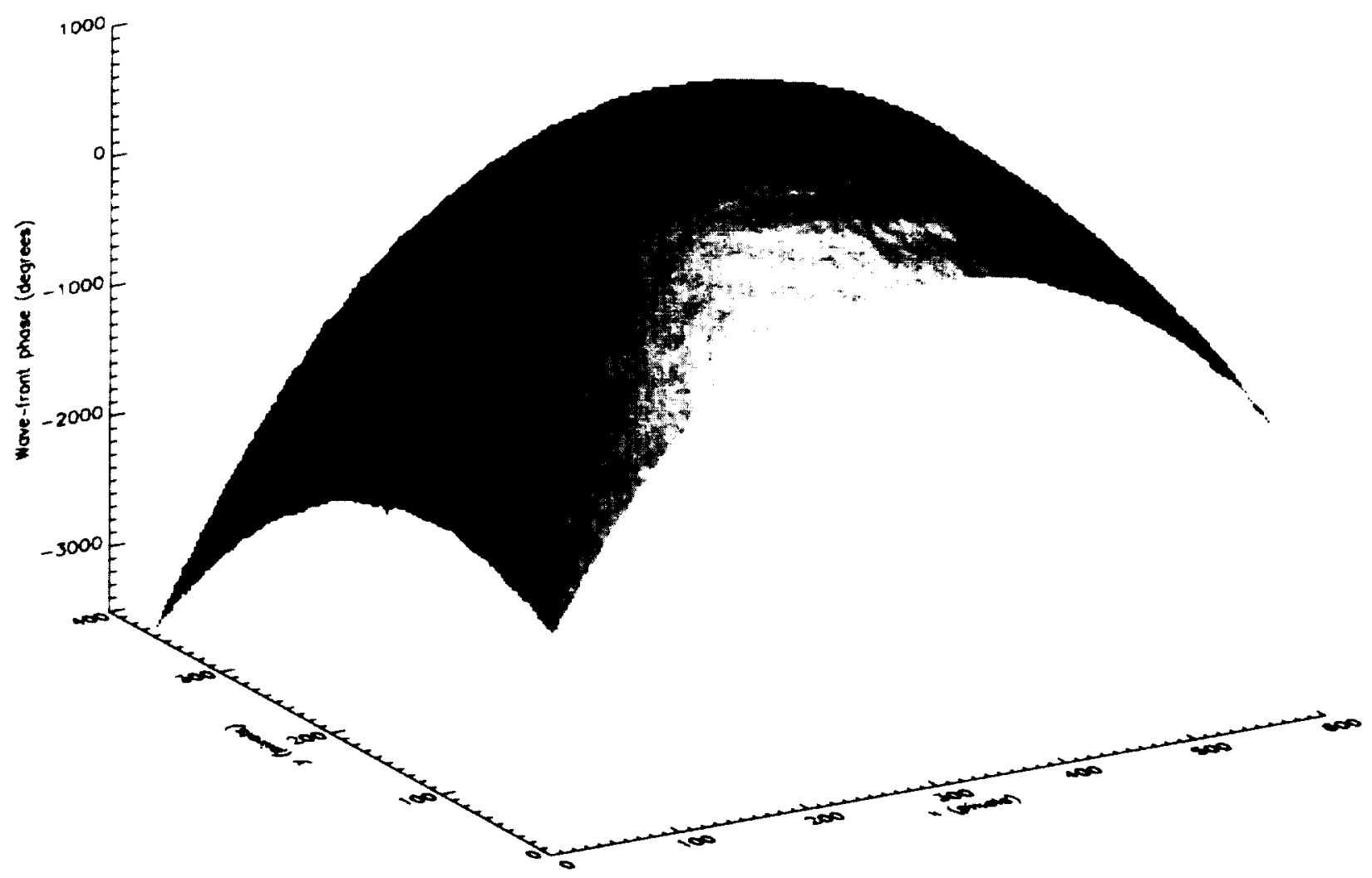

Fig. 10. Wave-front difference calculated with exact compensation algorithm [Eq. $99 \mid$.

five-frame algorithm. The shape is generally paraboloidal, but significant periodic error is present. Figure 10 shows the same wave front computed with the compensated five-frame algorithm, i.e., Eq. $|9|$. The periodic error, although not eliminated, is substantially reduced.

Figure 11 shows horizontal cross sections from the wave-front differences computed with each of the four methods described above, together with the corresponding cross section from the theoretical wave-front difference calculated from Eq. (11). These four methods are as follows: (a) polynomial fits to the wave fronts computed from the five-frame algorithm, (b) the five-frame algorithm, (c) the exact compensation algorithm $[$ Eq. (9) , and (d) the approxi-

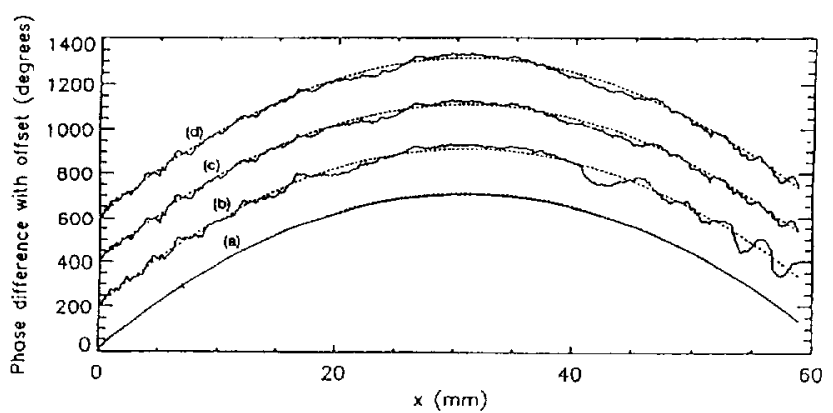

Fig. 11. Cross sections of wave-front differences computed from (a) two-dimensional polynomial curve fits, (b) standard five-frame

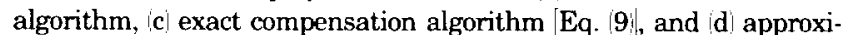
mate compensation algorithm $\mid \mathrm{Eq} .10$. Offsets are added for clarity. mate compensation algorithm $[\mathrm{Eq} .(10)]$. Offsets were added to each of these curves for clarity. It can be seen that in each case the shape of the wave-front difference is accurately measured, but the amount of periodic error depends on the computation method. The least error, not surprisingly, is obtained from the polynomial fits, Fig. 11(a), but this method retains the least information in the measured wave fronts as well. The standard five-frame algorithm, Fig. 11 b), produces the most error. Both the exact and the approximate compensation algorithms (c) and (d) provide significant improvement over the standard five-frame algorithm. These two compensation algorithms produce similar results because the object beam was much stronger than the reference beam. The amount of periodic error is reduced by these intensity compensation algorithms but not eliminated. The compensation is not perfect because the estimate of the object beam intensity distribution is not perfect for each frame. However, the overall shape of the wave front is being accurately measured.

\section{Conclusion}

The liquid-crystal point diffraction interferometer combines the robust, common-path design of the PDI with a simple method of optical phase control. The result is a compact new instrument for the measurement of optical wave fronts that uses phase-stepping interferometry for high data density and automatic data reduction. 
The LCPDI was used to measure a focus shift to demonstrate that the instrument can accurately measure wave-front differences. Because the LCPDI itself introduces aberrations into the interferograms, it is best used in applications in which wave-front differences are of interest. The difference operation will automatically compensate for the induced aberrations. Nonetheless, the instrument has potential for testing optical elements such as lenses and mirrors, provided that either a reference optic is available or the initial aberrations can be quantified.

The LCPDI currently modulates both the intensity and phase of the object beam. The unwanted intensity modulation causes a periodic error in the measured wave-front phase, so a normalization algorithm was developed to remove this error. The algorithm can completely eliminate the error if the object beam intensity distribution is accurately known for each phase step. If the object beam intensity is only approximated, then the algorithm still greatly attenuates the error. An approximate normalization algorithm was also presented; it requires fewer computational steps than the exact algorithm. These algorithms are useful for any phase-stepping interferometer in which the intensity of one beam is not constant from frame to frame.

We expect that the LCPDI will become a useful tool for providing automated data acquisition and reduction, with very high data density, for applications requiring a compact, inexpensive, robust interferometer.

\section{References}

1. V. P. Linnik, "Simple interferometer for the investigation of optical systems," C. R. Acad. Sci. USSR, 1, 208-210 (1933).

2. R. N. Smartt and J. Strong, "Point-diffraction interferometer," J. Opt. Soc. Am. 62, 737 (abstract only), (1972).

3. R. N. Smartt and W. H. Steel, "Theory and application of point-diffraction interferometers," Jpn. J. Appl. Phys. 14 (Suppl. 14-1), 351-357 (1975).

4. R. J. Speer, M. Crisp, D. Turner, S. Mrowka, and K. Tregidjo, "Grazing incidence interferometry: the use of the Linnik interferometer for testing image-forming reflection systems," Appl. Opt. 18, 2003-2012 (1979).

5. J. P. Marioge, B. Bonino, F. Bridou, P. Fournet, and M. Mullot, "La fabrication et le controle de surface toriques," J. Opt. (Paris) 15, 286-292 (1984).

6. C. Koliopoulos, O. Kwon, R. Shagam, J. C. Wyant, C. R. Hayslett, "Infrared point-diffraction interferometer," Opt. Lett. 3, 118-120 (1978).

7. W. Harris, S. Mrowka, and R. J. Speer, "Linnik interferometer: its use at short wavelengths," Appl. Opt. 21, 1155 (1982).

8. A. K. Aggarwal and S. K. Kaura, "Further applications of point diffraction interferometer," J. Opt. (Paris) 17, 135-138 (1986).
9. M. Giglio, E. Paganini, and U. Perini, "A self-aligning point diffraction interferometer for fluid studies," in Optical Components and Systems, A. Masson, ed., Proc. Soc. Photo-Opt. Instrum. Eng. 805, 82-86 (1987).

10. S. Musazzi, U. Perini, and F. Trespidi, "Point diffraction interferometer for fluids study in microgravity environment," Exp. Therm. Fluid Sci. 6, 49-55 1993).

11. K. Creath, "Phase-measurement interferometry techniques," in Progress in Optics XXVI, E. Wolf, ed. Elsevier, New York, 1988), Chap. 5.

12. K. Underwood, J. C. Wyant, and C. L. Koliopoulos, "Selfreferencing wavefront sensor," in Wavefront Sensing, N. Bareket and C. L. Koliopoulos, eds., Proc. Soc. Photo-Opt. Instrum. Eng. 351, 108-114 (1982).

13. O. Y. Kwon, "Multichannel phase-shifted interferometer," Opt. Lett. 9, 59-61 (1984).

14. H. Kadono, N. Takai, and T. Asakura, "New common-path phase shifting interferometer using a polarization technique," Appl. Opt. 26, 898-904 (1987).

15. J. Ojeda-Castaneda, Optical Shop Testing, 2nd ed., D. Malacara, ed. (Wiley, New York, 1992), Chap. 8.

16. H. Kadono, M. Ogusu, and S. Toyooka, "Phase shifting common path interferometer using a liquid-crystal phase modulator," Opt. Commun. 110, 391-400 (1994).

17. K. Iwata and T. Nishikawa, "Profile measurement with a phase-shifting common-path polarization interferometer," in Laser Interferometry: Quantitative Analysis of Interferograms: Third in a Series, R. J. Pryputniewicz, ed., Proc. Soc. Photo-Opt. Instrum. Eng. 1162, 389-394 (1990).

18. E. G. Churin and A. G. Sedukhin, "Diffraction common-path interferometer," Optoelectron. Instrum. Data Proc. 3, 71-74 (1994).

19. C. R. Mercer and K. Creath, "Liquid crystal point diffraction interferometer," Opt. Lett. 19, 916-918 (1994).

20. S.-T. Wu, U. Efron, and L. D. Hess, "Rotatory power and optical modulation of liquid crystals," in Spatial Light Modulators and Applications I, U. Efron, ed., Proc. Soc. Photo-Opt. Instrum. Eng. 465, 66-73 (1984).

21. J. Amako and T. Sonehara, "Kinoform using an electrically controlled birefringent liquid-crystal spatial light modulator," Appl. Opt. 30, 4622-4628 (1991).

22. C. R. Mercer and K. Creath, "Phase measurement using a liquid crystal point diffraction interferometer," in Industrial Optical Sensors for Metrology and Inspection, K. G. Harding and H. P. Stahl, eds., Proc. Soc. Photo-Opt. Instrum. Eng. 2349, 95-99 (1994).

23. A. E. Perregaux, J. F. Stephany, E. C. Faucz, R. A. Hudson, V. J. Hull, A. I. LaKatos, R. A. Martel, R. Narang, and B. Richter, "Transient nematic liquid crystal image bar for electrophotographic printers,"SID Dig. 28, 360-362 (1987).

24. G. H. Heilmeier, J. A. Castellano, and L. A. Zanoni, "Guesthost interactions in nematic liquid crystals," Mol. Cryst. Liq. Cryst. 8, 293-304 (1969).

25. C. R. Mercer, "Liquid crystal point diffraction interferometer," Ph.D. dissertation (University Microfilms, Inc., Tueson Ariz., 1995).

26. J. D. Gaskill, Linear Systems, Fourier Transforms, and Optics (Wiley, New York, 1978). 